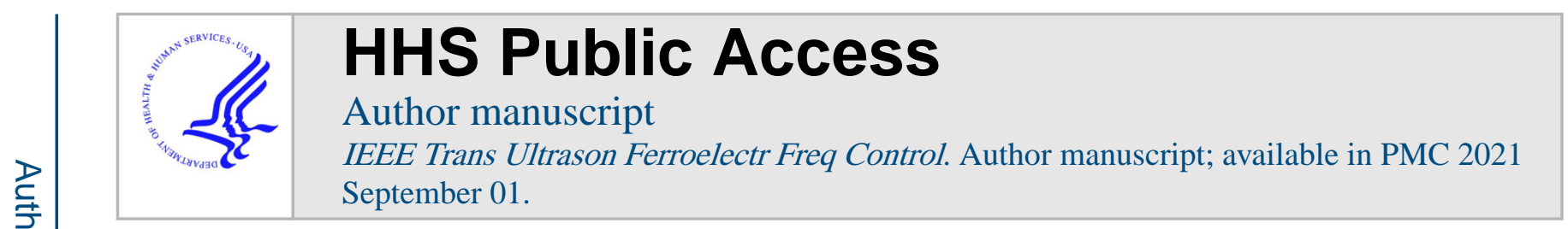

Published in final edited form as:

IEEE Trans UItrason Ferroelectr Freq Control. 2020 September ; 67(9): 1848-1853. doi:10.1109/

TUFFC.2020.2985369.

\title{
Transparent High-Frequency Ultrasonic Transducer for Photoacoustic Microscopy Application
}

\author{
Ruimin Chen", \\ Department of Biomedical Engineering, Viterbi School of Engineering, University of Southern \\ California, Los Angeles, CA 90089 USA, and the USC Roski Eye Institute, Keck School of \\ Medicine, University of Southern California, Los Angeles, CA 90089 USA \\ Yun $\mathrm{He}^{\#}$, \\ Caltech Optical Imaging Laboratory, Andrew and Peggy Cherng Department of Medical \\ Engineering, Department of Electrical Engineering, California Institute of Technology, Pasadena, \\ CA 91125 USA
}

Department of Biomedical Engineering, Washington University in St Louis, St Louis, MO 63130 USA

Junhui Shi,

Caltech Optical Imaging Laboratory, Andrew and Peggy Cherng Department of Medical Engineering, Department of Electrical Engineering, California Institute of Technology, Pasadena, CA 91125 USA

\section{Christopher Yung,}

Applied Physics Division, National Institute of Standards and Technology, Boulder, CO 80305, USA

\section{Jeeseong Hwang,}

Applied Physics Division, National Institute of Standards and Technology, Boulder, CO 80305, USA

\author{
Lihong V. Wang [Fellow, IEEE], \\ Caltech Optical Imaging Laboratory, Andrew and Peggy Cherng Department of Medical \\ Engineering, Department of Electrical Engineering, California Institute of Technology, Pasadena, \\ CA 91125 USA

\section{Qifa Zhou [Fellow, IEEE]} \\ Department of Biomedical Engineering, Viterbi School of Engineering, University of Southern \\ California, Los Angeles, CA 90089 USA, and the USC Roski Eye Institute, Keck School of \\ Medicine, University of Southern California, Los Angeles, CA 90089 USA \\ \# These authors contributed equally to this work.
}

Corresponding authors: Lihong V. Wang; Qifa Zhou. 1vw@caltech.edu, qifazhou@usc.edu.

C. Yung and J. Hwang are with the Applied Physics Division, National Institute of Standards and Technology, Boulder, CO 80305, USA. Certain commercial equipment, instruments, or materials are identified in this paper in order to specify the experimental procedure adequately. Such identification is not intended to imply recommendation or endorsement by the National Institute of Standards and Technology, nor is it intended to imply that the materials or equipment identified are necessarily the best available for the purpose. 


\section{Abstract}

We report the development of an optically transparent high-frequency ultrasonic transducer using lithium niobate single-crystal and indium-tin-oxide electrodes with up to $90 \%$ optical transmission in the visible to near infrared spectrum. The center frequency of the transducer was at 36.9 $\mathrm{MHz}$ with $33.9 \%$, at $-6 \mathrm{~dB}$ fractional bandwidth. Photoacoustic imaging capability of the fabricated transducer was also demonstrated by successfully imaging a resolution target and mouse ear vasculatures in vivo, which were irradiated by a $532 \mathrm{~nm}$ pulse laser transmitted through the transducer.

\section{Keywords}

Transparent ultrasonic transducer; high-frequency ultrasonic transducer; photoacoustic microscopy

\section{Introduction}

PHOTOACOUSTIC microscopy (PAM) remains the prime choice for label-free microscopic imaging of the optical absorption contrast in tissue [1-3]. It usually employs a single highfrequency ultrasonic transducer to acquire the acoustic waves converted from absorbed optical energy via transient thermoelastic expansion [4-6]. So far, the most widely used PAMs for in vivo imaging applications utilize a reflection-mode setup: both the optical illumination and ultrasonic detection placed on the same side of the imaging object [7-11]. However, it imposes certain constraints on PAM's performance. A long working distance is usually required to accommodate the sizeable transducer and the optical-acoustic splitter, thus limiting PAM's numerical aperture and resulting in a poor lateral resolution [12, 13]. Transmission-mode PAMs have achieved finer lateral resolutions, but they are mainly limited to imaging ex vivo samples [14, 15]. Further, the complex optical-acoustic splitter, two prisms sandwiching a thin layer of silicon oil, is challenging to make and maintain [12, 13].

To address this problem, doughnut-shaped transducers with a central opening for light passage have been introduced to reduce the obstruction in the optical path $[7,10,16]$. But its size is still too big for PAM to achieve a resolution comparable to optical microscopy [16]. Taking another approach, thin-film transducers that are optically transparent offer a desirable solution. Transparent optic-based ultrasonic detectors include Fabry-Perot etalons [17, 18], micro-ring resonators [19-22], among others [23]. However, they require additional optical instruments such as probe lasers and detectors. Further, it is difficult to integrate these detectors into conventional ultrasound emission devices for ultrasonic/photoacoustic dualmodality imaging. Optically transparent piezoelectric-based devices have been introduced to circumvent this problem. Such devices include ultrasonic transducers with indium-tin-oxide (ITO) coated polyvinylidene fluoride (PVDF) film [24-26], but these PVDF films only achieved a transmittance of 0.8 at best at $532 \mathrm{~nm}$. Also, PVDF's coupling coefficient $\left(k_{\mathrm{t}}\right)$ is not comparable with other popular piezoelectric materials. Another type is capacitive micromachined ultrasonic transducer (CMUT) [27]. This device has limited operating frequency (<20 MHz) and is of high cost from specialized micro-electro-mechanical systems (MEMS) fabrication techniques. 
Moreover, transducers operating at the range of $30-50 \mathrm{MHz}$ are favorable for PAM imaging, since the frequency spectrum of photoacoustic waves after propagating through tissue mainly resides in this range [8]. Therefore, there is a need to find a suitable material for high-frequency ultrasonic transducers with high optical transmission for reflectance mode PAM applications.

Among known piezoelectric materials, lithium niobate $\left(\mathrm{LiNbO}_{3}\right)$ piezoelectric material possesses several properties that are ideal to meet the need. It is a single-crystal material that displays a flat transmittance of 0.8 at a broad range of wavelengths between $350 \mathrm{~nm}$ and $5200 \mathrm{~nm}$ [28]. It provides good electromechanical coupling, low dielectric constant, and high longitudinal sound speed, ideal for large aperture high sensitivity and high-frequency optically transparent transducers. A brief list of the material properties for $\mathrm{LiNbO}_{3}$ can be found in [29]. Brodie et al. developed an optically transparent piezoelectric transducer with ITO coated $\mathrm{LiNbO}_{3}$ for ultrasonic particle manipulation [30]. Recently, Dangi et al. reported a promising $\mathrm{LiNbO}_{3}$-based transparent ultrasonic transducer operating at $14.5 \mathrm{MHz}$ for PA imaging [31].

The purpose of this study is to develop a transducer for PAM applications with a high optical transmission (> $80 \%$ ) and a high frequency (> $30 \mathrm{MHz}$ ) response. The high transmission of $\mathrm{LiNbO}_{3}$ single-crystal substrate coated with ITO thin films on both sides is demonstrated over a broad range of wavelengths $(450 \mathrm{~nm}-1064 \mathrm{~nm})$. PAM imaging performance of the transparent transducer is evaluated by imaging a carbon nanotube-based resolution target and mouse ear vasculatures in vivo.

\section{Materials and methods}

\section{A. Transducer Design}

Parylene thin-film (Parylene C, Specialty Coating Systems, Indianapolis, IN, USA) with nominal acoustic impedance of 2.5 MRayl was selected as the material for the matching layer because it exhibits very little absorption in the visible region and is, therefore, transparent and colorless. To facilitate transducer transparency, an insulating and optically transparent epoxy (EPO-TEK 301, Epoxy Technology, Inc., Billerica, MA, USA) with nominal acoustic impedance of 3.05 MRayl was chosen as the material for backing.

Design parameters of the transducer are summarized in Table I. The inner structure cross section schematic of the transducer is shown in Fig. 1(a). A Krimboltz, Leedom, and Mattaei (KLM) transducer equivalent circuit model-based modeling software (PiezoCAD, Sonic Concepts, Woodinville, WA, USA) was used to simulate and optimize the transducer design [32].

\section{B. Transducer Fabrication}

A $36^{\circ}$ rotated $\mathrm{Y}$-cut $\mathrm{LiNbO}_{3}$ wafer with a dimension of $1 \mathrm{~cm} \times 1 \mathrm{~cm}$ and a thickness of 100 $\mu \mathrm{m}(\lambda / 2)$ was acquired from Boston Piezo-optics Inc. (Bellingham, MA, USA) to make a transducer operating at $37 \mathrm{MHz}$. High optical transmission with surface electrodes was achieved by ITO coatings. Both top and bottom sides of the $\mathrm{LiNbO}_{3}$ wafer were electroded by sputtering ITO thin films of approximately $108 \mathrm{~nm}$ in total thickness. ITO was DC 
magnetron sputtered from a $50 \mathrm{~mm}$ diameter ITO target $\left(\mathrm{In}_{2} \mathrm{O}_{3} / \mathrm{SnO}_{2}\right.$ 90/10 wt \%, Kurt J. Lesker Company, Jefferson Hills, PA, USA) at $20 \mathrm{~W}, 933 \mathrm{~Pa}$ (7 mTorr, Argon flow $\approx 30$ $\mathrm{cm}^{3} / \mathrm{min}$ ) for 80 minutes. The deposition rate was monitored using a quartz crystal microbalance $(\mathrm{QCM})$ with a measured rate of $0.03 \mathrm{~nm} / \mathrm{s}$ (density $=7.14 \mathrm{~g} / \mathrm{cm}^{3}$, z-ratio $=1$, tooling $=100 \%)$. For an accurate measure of on-sample deposition rate $\left(\mathrm{LiNbO}_{3}\right.$ crystals and QCM are at differing heights), ITO was sputtered on a silicon substrate under similar conditions for $20 \mathrm{~min}$ and measured using a white light spectrometer (Filmetrics F40, KLA, Milpitas, CA, USA). The 20 min sputtered film yielded a refractive index of 1.89 and a thickness of $27 \mathrm{~nm}$. Extrapolating these values, we estimate the thickness of the ITO film on the $\mathrm{LiNbO}_{3}$ to be $108 \mathrm{~nm}$. A hook-up wire (Gauge 24, McMaster-Carr Supply Company, Santa Fe Springs, CA, USA) consisting of stranded copper wires was connected to the bottom ITO electrode on the edge of the $\mathrm{LiNbO}_{3}$ wafer with a small amount of conductive epoxy (E-SOLDER 3022, Von Roll Isola Inc., New Haven, CT, USA). A home-made brass housing with an inner opening of $15 \mathrm{~mm}$ in diameter and a subminiature version A (SMA) connector on the side was placed concentrically with the $\mathrm{LiNbO}_{3}$ wafer. The other end of the stranded copper wire was connected to the SMA connector. Degassed epoxy (EPO-TEK 301) with a total weight of $0.2 \mathrm{~g}$ was then poured into the brass housing to achieve a $1 \mathrm{~mm}$ thick epoxy after curing. For ground connection, a small amount of E-SOLDER 3022 was applied at the four corners on the front surface of the $\mathrm{LiNbO}_{3}$ wafer to connect the top ITO electrode with brass housing. Finally, a $16 \mu \mathrm{m}$ thick $(\lambda / 4)$ parylene thin-film (Parylene C, Specialty Coating Systems, Indianapolis, IN, USA) layer was vapor-deposited onto the external surface of the transducer by a parylene coater (PDS 2010, Specialty Coating Systems, Indianapolis, IN, USA). A photograph of the fabricated transducer is shown in Fig. 1(b).

\section{PAM Imaging}

To demonstrate applications in PAM, we conducted phantom and animal imaging with a reflection-mode PAM [Fig. 2(a)]. The laser beam with $4 \mathrm{kHz}$ repetition rate from a $532 \mathrm{~nm}$ picosecond pulsed laser (APL-4000-1064, Attodyne Inc., Toronto, ON, Canada) was focused onto the imaging objects by an aspherical lens (AL2550-A, Thorlabs Inc., Newton, NJ, USA; $50 \mathrm{~mm}$ focal length, $0.23 \mathrm{NA}$ ), through the transparent transducer. Excited PA waves were detected by the transparent transducer, which was placed in a tank of deionized water for acoustic coupling. A data acquisition unit (ATS9350, AlazarTech, Inc., Montreal, QC, Canada) was used to acquire the PA signal at a 200-MHz sampling rate. The imaging objects were mounted on a two-axis translation stage (PLS-85, PI miCos GmbH, Auburn, MA, USA), and it took $\sim 4 \mathrm{~min}$ to acquire an image of $1 \mathrm{~mm} \times 0.8 \mathrm{~mm}$ region, with a scanning step size of $3.1 \mu \mathrm{m}$. Maximum amplitude projection (MAP) images were produced in post-processing by taking the peak-to-peak amplitude of each PA A-line signal.

\section{Results}

\section{A. Transducer Performance Evaluation}

First, we evaluated the transducer's optical transmission efficiency. An optical parametric oscillator laser (NT242, EKSPLA, Vilnius, Lithuania) provided the illumination at a broad spectrum, and the ratio of the laser light transmitted through the transducer was measured by 
a laser power meter (Vega, Ophir Optronics Solutions Ltd., Jerusalem, Israel). Transmission efficiencies greater than $80 \%$ from $450 \mathrm{~nm}$ to $1064 \mathrm{~nm}$ were measured [Fig. 2(b)]. The trough near $700 \mathrm{~nm}$ was also observed on the $\mathrm{LiNbO}_{3}$-based transducer in [30].

Electrical impedance and phase angle of the transducer was measured by an impedance analyzer (HP 4294A, Agilent Technologies, Santa Clara, CA, USA) and shown in Fig. 3(b). The resonance frequency $\left(f_{\mathrm{r}}\right)$ and anti-resonance frequency $\left(f_{\mathrm{a}}\right)$ of the transducer were measured to be $33.9 \mathrm{MHz}$ and $40.3 \mathrm{MHz}$, respectively. Using the IEEE standard on piezoelectricity [33], the effective electromechanical coupling coefficient ( $\left.k_{\text {eff }}\right)$ of the transducer was calculated to be 0.54 . It was observed that the measured results are very close to the PiezoCAD simulation results $\left(f_{\mathrm{r}}=31.0 \mathrm{MHz}, f_{\mathrm{a}}=37.8 \mathrm{MHz}\right.$, and $\left.k_{\text {eff }}=0.57\right)$ as shown in Fig. 3(a). The electrical impedance of the transducer was measured as $161 \Omega$ to $258 \Omega$ around the resonance frequency of the transducer, while the PiezoCAD simulation from $7 \Omega$ to $22 \Omega$. The difference between simulation and measurement especially for electrical impedance could be contributed by the sheet resistance which cannot be simulated in PiezoCAD.

The pulse-echo response of the transducer was measured and analyzed by the method described in [34]. The transducer was mounted on a holder and immersed in a tank filled with deionized water. An X-cut quartz plate was placed about $5 \mathrm{~mm}$ away from the transducer as a reflector. The transducer was excited by a pulser-receiver (Panametrics 5900PR, Olympus NDT Inc., Waltham, MA, USA) with $1 \mu \mathrm{J}$ energy per pulse, $200 \mathrm{~Hz}$ pulse repetition rate, and $50 \Omega$ damping factor. The received pulse-echo response and its computed frequency spectrum are shown in Fig. 4(b). The center frequency of the transducer was calculated to be $36.9 \mathrm{MHz}$, and the $-6 \mathrm{~dB}$ bandwidth was $33.9 \%$. Peak-to-peak received voltage $\left(V_{\mathrm{p}-\mathrm{p}}\right)$ of the unamplified pulse-echo signal was $0.45 \mathrm{~V}$, indicating the transducer has quite high sensitivity compared to other published transparent transducers [25, 30, 31]. The measured center frequency derived from the pulse-echo result is in good agreement with the PiezoCAD simulation (35.6 MHz) [Fig. 4(a)].

\section{B. PAM Imaging with the Transparent Ultrasonic Transducer}

Fig. 5(a) shows the MAP image of a resolution target phantom that is composed of carbon nanotube patterns transferred onto a polydimethylsiloxane substrate [35]. The smallest patterns, periodic lines of $10 \mu \mathrm{m}$ width, $100 \mu \mathrm{m}$ long, and $40 \mu \mathrm{m}$ pitch were clearly resolved, while the axial resolution is calculated to be $105 \mu \mathrm{m}$ [36]. The laser pulse energy irradiated on the target was measured to be $\sim 200 \mathrm{~nJ}$, resulting in signal-to-noise ratio (SNR) of $\sim 41.5$. Further, we demonstrated the transparent transducer's in vivo imaging capability by imaging the mouse ear at the optical wavelength of $532 \mathrm{~nm}$. At this wavelength, the hemoglobin in red blood cells (RBCs) is the primary absorber in tissue, thus enabling imaging the vascular anatomy. The laboratory animal protocols were approved by the Institutional Animal Care and Use Committee of California Institute of Technology. Fig. 5(b) shows the MAP image of a $1 \mathrm{~mm} \times 0.8 \mathrm{~mm}$ area of the mouse ear. It can be seen that vessels of a diameter as small as $30 \mu \mathrm{m}$ were clearly imaged. 


\section{Conclusions and Discussions}

In this work, a transparent high-frequency ultrasonic transducer using ITO coated $\mathrm{LiNbO}_{3}$ single-crystal was developed for PAM application. The fabricated transducer exhibited an effective electromechanical coupling coefficient $k_{\text {eff }}$ of 0.54 , a center frequency of 36.9 MHz, a $-6 \mathrm{~dB}$ bandwidth of $33.9 \%$, a high sensitivity of $0.45 \mathrm{~V}$ peak-to-peak received voltage $\left(V_{\mathrm{p}-\mathrm{p}}\right)$, as well as an excellent transparency of up to $90 \%$ in the visible range. Imaging capability of the transducer for PAM was also demonstrated by successfully imaging a resolution target and mouse ear vasculatures in vivo, with the laser beam transmitting directly through the transducer. These results will inspire further development of high-frequency ultrasonic transducers in transparent form, which will enable PAM with finer resolution.

Recently, there are quite a few reports on piezoelectric-based transparent ultrasonic transducers fabricated with various techniques [24, 26, 30, 31, 37]. However, this work stands out in the following ways: 1) the transducer's frequency response is the highest, and best matches the frequency spectrum of photoacoustic waves, mainly at the $30-50 \mathrm{MHz}$ range, in in vivo imaging applications. 2) in vivo PAM imaging capability was first demonstrated by this transducer due to its higher sensitivity over others.

Although the transducer demonstrated very good resolution on imaging the resolution target. However, it was not capable to resolve microvessels in the mouse vasculature imaging experiment due to insufficient sensitivity of the transducer. Therefore, there is room for improvement in the performance of transducer. Firstly, the transducer has a relatively high electrical impedance due to high sheet resistance of the ITO thin-film electrodes compared with conventional Au thin-film electrodes [38] which affected transducer sensitivity. It can be improved by choosing a transparent electrode material with lower resistivity. Secondly, choosing suitable materials for both matching layer and backing in ultrasonic transducers can compensate the large acoustic impedance mismatch between piezoelectric element and the load which is a key factor that limits the performance of transducers in terms of sensitivity and bandwidth [39]. Parylene and Epotek-301, both have low acoustic impedance, were chosen as the matching layer material and backing material in the current transducer design, respectively. They limited $-6-\mathrm{dB}$ bandwidth of the transducer and degraded transducer sensitivity. Therefore, it is necessary to investigate transparent materials with proper acoustic impedance for the matching layer and the backing in order to improve both sensitivity and bandwidth of the transducer. Furthermore, an optimal front matching scheme incorporating two or more matching layers will also need to be studied.

Employing focusing techniques on the transducer such as using an acoustic lens can also enhance transducer sensitivity. However, focused transducer was not preferred in this work because conventional PAM imaging systems incorporating focused transducers require not only confocal alignment between laser light illumination and acoustic detection, but also mechanical scan of either the transducer or the imaging subjects, which significantly limits PA imaging speed. The next goal of this work is to develop a fast, compact, wearable or hand-holdable PAM imaging device [40], which has broad applications in both in vivo biomedical studies and patient monitoring in clinics. A stationary unfocused transparent 
transducer, requiring only optical scanning for PAM imaging, has an enormous potential for achieving this goal.

\section{Acknowledgments}

This work was supported (in part) by the US National Institute of Health under Grant 1R01EY026091.

\section{Biography}

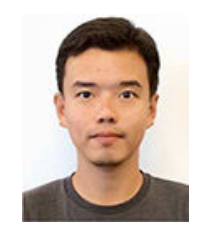

Ruimin Chen received his B.S. degree in Biomedical Engineering from University of Electronics Science and Technology of China, Chengdu, China in 2006, and both his M.S. and his Ph.D. degrees in Biomedical Engineering from University of Southern California, Los Angeles, CA, in 2008 and 2014, respectively. He worked as a postdoctoral research associate and resource manager in the NIH Ultrasonic Transducer Resource Center at the Department of Biomedical Engineering of University of Southern California. Currently, he is an ultrasound sensor engineer at Masimo Corporation, Irvine, CA.

His research interests include the design, modeling, and fabrication of high-frequency ultrasonic transducers and arrays for medical imaging applications, piezoelectric material characterization, and photoacoustic imaging.

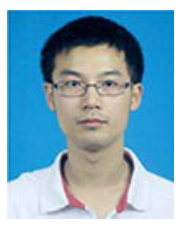

Yun He received his Ph.D. degree in Biomedical Engineering from Washington University in St. Louis, St Louis, MO in 2019. He also received his B.S. and M.S. degrees in Biomedical Engineering from Tsinghua University, Beijing, China.

His research focuses on the development and application of optical-resolution photoacoustic microscopy.

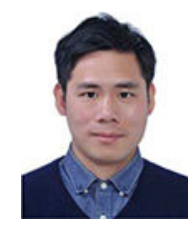

Junhui Shi received his B.S. in chemical physics from the University of Science and Technology of China, Hefei, China. Then, he continued to study chemistry and received his Ph.D. at Princeton University, Princeton, NJ. He was working on theoretical chemical 
dynamics and experimental nuclear magnetic resonance spectroscopy. Afterward, he worked on photoacoustic imaging in the Department of Biomedical Engineering of Washington University in St. Louis, St. Louis, MO, and then in the Department of Medical Engineering of Caltech, Pasadena, CA. Currently, he is a senior researcher at Zhejiang Lab, Hangzhou, China.

His research interests include photoacoustic microscopy, photoacoustic tomography, nuclear magnetic imaging, and their applications in biomedical studies.

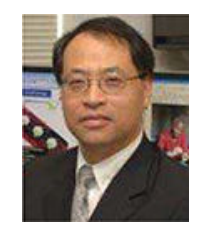

Jeeseong Hwang is a research biophysicist at Physical Measurement Laboratory of the US National Institute of Standards and Technology (NIST), Boulder, CO, conducting research on measurement science for quantitative biophysics and optical medical imaging standards. Before joining NIST, he did research on nanoscale bio-imaging at the Johns Hopkins University, Baltimore, MD, jointly with the AT\&T Bell Laboratories. He has served in IEEE, SPIE, and ISO and other international conferences and organizations.

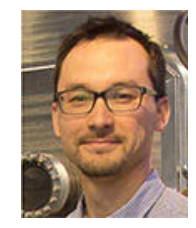

Christopher Yung received his B.S. degree from Texas A\&M University, College Station, Texas and his Ph.D. degree from the University of California, Santa Barbara, CA, in 1997 and 2004, respectively, both in physics. He is currently a physicist in the Applied Physics Division at the National Institute of Standards and Technology, Boulder, CO.

His research interests include microbolometers, carbon nanotube devices, microfabrication and IBAD epitaxial coatings for flexible electronics ( $\mathrm{GaN}$, superconductors, etc.).

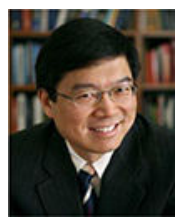

Lihong V. Wang is Bren Professor of Medical and Electrical Engineering at Caltech. Published 530 journal articles (h-index $=134$, citations $=75,000$ ). Delivered 5350 keynote/ plenary/invited talks. Published the first functional photoacoustic CT, 3D photoacoustic microscopy, and compressed ultrafast photography (world's fastest camera). Served as Editor-in-Chief of the Journal of Biomedical Optics. Received the Goodman Book Award, NIH Director's Pioneer Award, OSA Mees Medal, IEEE Technical Achievement and Biomedical Engineering Awards, SPIE Chance Biomedical Optics Award, IPPA Senior 
Prize, OSA Feld Biophotonics Award, and an honorary doctorate from Lund University, Sweden. Inducted into the National Academy of Engineering.

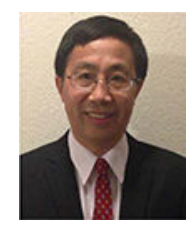

Qifa Zhou received his Ph.D. degree from the Department of Electronic Materials and Engineering at Xi' an Jiaotong University, China in 1993. He is currently a professor of Biomedical Engineering and Ophthalmology at the University of Southern California (USC), Los Angeles, CA. Before joining USC in 2002, he worked in the Department of Physics at Sun Yat-Sen University, Guangzhou, China, the Department of Applied Physics at Hong Kong Polytechnic University, Hong Kong, China, and the Materials Research Laboratory at Pennsylvania State University, State College, PA.

Dr. Zhou is a fellow of the Institute of Electrical and Electronics Engineers (IEEE), the International Society for Optics and Photonics (SPIE), and the American Institute for Medical and Biological Engineering (AIMBE). He is also a member of the Technical Program Committee of the IEEE International Ultrasonics Symposium, and is an Associate Editor of the IEEE Transactions on Ultrasonics, Ferroelectrics, and Frequency Control. His research focuses on the development of piezoelectric high-frequency ultrasonic transducers for biomedical ultrasound and photoacoustic imaging, including intravascular imaging, cancer imaging, and ophthalmic imaging. He is also actively exploring ultrasonic mediated therapeutic technology including trans-sclera drug delivery, as well as ultrasound for retinal and brain stimulation. He has published more than 200 peer-reviewed articles in journals including Nature Medicine, Advanced Materials, and Progress in Materials Science.

\section{References}

[1]. Wang LV and Yao J, "A practical guide to photoacoustic tomography in the life sciences," Nat. Methods, vol. 13, no. 8, pp. 627-638, Jul. 2016. [PubMed: 27467726]

[2]. Wang LV and Hu S, "Photoacoustic tomography: in vivo imaging from organelles to organs," Science, vol. 335, no. 6075, pp. 1458-1462, Mar. 2012. [PubMed: 22442475]

[3]. Ntziachristos V, "Going deeper than microscopy: the optical imaging frontier in biology," Nat. Methods, vol. 7, no. 8, pp. 603-614, Aug. 2010. [PubMed: 20676081]

[4]. Yao J and Wang LV, "Sensitivity of photoacoustic microscopy," Photoacoustics, vol. 2, no. 2, pp. 87-101, Apr. 2014. [PubMed: 25302158]

[5]. Wang LV and Wu H-I, Biomedical Optics: Principles and Imaging. Hoboken, NJ, USA: Wiley, 2012.

[6]. Wang LV, “Tutorial on photoacoustic microscopy and computed tomography,” IEEE J. Sel. Top. Quantum Electron, vol. 14, no. 1, pp. 171-179, Jan-Feb 2008.

[7]. Lan B et al., "High-speed widefield photoacoustic microscopy of small-animal hemodynamics," Biomed. Opt. Express, vol. 9, no. 10, pp. 4689-4701, Sep. 2018. [PubMed: 30319896]

[8]. Yao J et al., "High-speed label-free functional photoacoustic microscopy of mouse brain in action,” Nat. Methods, vol. 12, no. 5, pp. 407-410, Mar. 2015. [PubMed: 25822799]

[9]. Wang L, Maslov K, and Wang LV, "Single-cell label-free photoacoustic flowoxigraphy in vivo," Proc. Natl. Acad. Sci. U.S.A, vol. 110, no. 15, pp. 5759-5764, Apr. 2013. [PubMed: 23536296] 
[10]. Wang T et al., "Multiparametric photoacoustic microscopy of the mouse brain with $300-\mathrm{kHz}$ Aline rate," Neurophotonics, vol. 3, no. 4, art. 045006, pp. 1-6, Nov. 2016.

[11]. He Y et al., "In vivo label-free photoacoustic flow cytography and on-the-spot laser killing of single circulating melanoma cells," Sci. Rep, vol. 6, art. 39616, pp. 1-8, Dec. 2016. [PubMed: 28442746]

[12]. Hu S, Maslov K, and Wang LV, "Second-generation optical-resolution photoacoustic microscopy with improved sensitivity and speed," Opt. Lett, vol. 36, no. 7, pp. 1134-1136, Apr. 2011. [PubMed: 21479007]

[13]. Maslov K, Zhang HF, Hu S, and Wang LV, "Optical-resolution photoacoustic microscopy for in vivo imaging of single capillaries," Opt. Lett, vol. 33, no. 9, pp. 929-931, 5 2008. [PubMed: 18451942]

[14]. Shi J et al., "High-resolution, high-contrast mid-infrared imaging of fresh biological samples with ultraviolet-localized photoacoustic microscopy," Nat. Photonics, vol. 13, pp. 609-615, 52019. [PubMed: 31440304]

[15]. Zhang C et al., "Reflection-mode submicron-resolution in vivo photoacoustic microscopy," J. Biomed. Opt, vol. 17, no. 2, art. 020501, pp. 1-3, Feb. 2012.

[16]. Wong TT et al., "Label-free automated three-dimensional imaging of whole organs by microtomy-assisted photoacoustic microscopy," Nat. Commun, vol. 8, art. 1386, pp. 1-8, Nov. 2017. [PubMed: 28232747]

[17]. Zhang E and Beard P, "Broadband ultrasound field mapping system using a wavelength tuned, optically scanned focused laser beam to address a Fabry Perot polymer film sensor," IEEE Trans. Ultrason. Ferroelectr., Freq. Control, vol. 53, no. 7, pp. 1330-1338, Jul. 2006. [PubMed: 16889340]

[18]. Ansari R, Zhang E, Desjardins AE, Beard PC, "All-optical endoscopic probe for high resolution 3D photoacoustic tomography,” Proc. SPIE, vol. 10064, pp. 100641W-1-100641W-6, Mar. 2017.

[19]. Chen S-L, Ling T, Baac HW, Guo LJ, "Photoacoustic endoscopy using polymer microring resonators," Proc. SPIE, vol. 7899, pp. 78992T-1-78992T-6, Feb. 2011.

[20]. Li H, Dong B, Zhang Z, Zhang HF, and Sun C, "A transparent broadband ultrasonic detector based on an optical micro-ring resonator for photoacoustic microscopy," Sci. Rep, vol. 4, art. 4496, pp. 1-8, Mar. 2014.

[21]. Dong B et al., "Isometric multimodal photoacoustic microscopy based on optically transparent micro-ring ultrasonic detection," Optica, vol. 2, no. 2, pp. 169-176, Feb. 2015. [PubMed: 29805988]

[22]. Li H et al., "Disposable ultrasound-sensing chronic cranial window by soft nanoimprinting lithography," Nat. Commun, vol. 10, art. 4277, pp. 1-9, Sep. 2019. [PubMed: 30602773]

[23]. Chen S-L, Guo LJ, and Wang X, "All-optical photoacoustic microscopy," Photoacoustics, vol. 3, no. 4, pp. 143-150, Dec. 2015. [PubMed: 31467845]

[24]. Niederhauser JJ, Jaeger M, Hejazi M, Keppner H, and Frenz M, "Transparent ITO coated PVDF transducer for optoacoustic depth profiling," Opt. Commun, vol. 253, no. 4-6, pp. 401-406, Sep. 2005.

[25]. Blumenrother E, Melchert O, Kanngiesser J, Wollweber M, and Roth B, "Single transparent piezoelectric detector for optoacoustic sensingdesign and signal processing," Sensors, vol. 19, no. 9, art. 2195, pp. 1-11, 52019.

[26]. Fang C, Hu H, and Zou J, “A focused optically transparent PVDF transducer for photoacoustic microscopy,” IEEE Sens. J, vol. 20, no. 5, pp. 2313-2319, 32020.

[27]. Li Z, Ilkhechi AK, and Zemp R, "Transparent capacitive micromachined ultrasonic transducers (CMUTs) for photoacoustic applications," Opt. Express, vol. 27, no. 9, pp. 13204-13218, Apr. 2019. [PubMed: 31052849]

[28]. Chua SJ and Li B, Optical Switches: Materials and Design. ýAmsterdam, Netherlands: Elsevier Science, 2010.

[29]. Cannata JM, Ritter TA, Chen W-H, Silverman RH, and Shung KK, "Design of efficient, broadband single-element (20-80 MHz) ultrasonic transducers for medical imaging applications," IEEE Trans. Ultrason. Ferroelectr., Freq. Control, vol. 50, no. 11, pp. 1548-1557, Nov. 2003. [PubMed: 14682638] 
[30]. Brodie GWJ, Qiu Y, Cochran S, Spalding GC, and MacDonald MP, “Optically transparent piezoelectric transducer for ultrasonic particle manipulation," IEEE Trans. Ultrason. Ferroelectr., Freq. Control, vol. 61, no. 3, pp. 389-391, Mar. 2014. [PubMed: 24569243]

[31]. Dangi A, Agrawal S, and Kothapalli SR, "Lithium niobate-based transparent ultrasound transducers for photoacoustic imaging," Opt. Lett, vol. 44, no. 21, pp. 5326-5329, Nov. 2019. [PubMed: 31674999]

[32]. Krimholtz R, Leedom DA, and Matthaei GL, "New equivalent circuits for elementary piezoelectric transducers,” Electron. Lett, vol. 6, no. 13, pp. 398-399, Jun. 1970.

[33]. "Publication and proposed revision of ANSI/IEEE standard 176-1987 "ANSI/IEEE standard on piezoelectricity”, IEEE Trans. Ultrason. Ferroelectr., Freq. Control, vol. 43, no. 5, p. 717, Sep. 1996.

[34]. Chen R et al., "Eco-friendly highly sensitive transducers based on a new KNN-NTK-FM leadfree piezoelectric ceramic for high-frequency biomedical ultrasonic imaging applications," IEEE Trans. Biomed. Eng, vol. 66, no. 6, pp. 1580-1587, Jun. 2019. [PubMed: 30452346]

[35]. Hwang J. Development of photoacoustic phantoms towards quantitative evaluation of photoacoustic imaging devices; Presented at SPIE Photonics West 2018; 10494-77. Paper

[36]. Zhang C, Maslov K, Yao JJ, and Wang LHV, "In vivo photoacoustic microscopy with 7.6-mu m axial resolution using a commercial 125-MHz ultrasonic transducer," Journal of Biomedical Optics, vol. 17, no. 11, art. 116016, pp. 1-6, Nov. 2012.

[37]. Chen $\mathrm{H}$ et al., "Optical-resolution photoacoustic microscopy using transparent ultrasound transducer," Sensors, vol. 19, no. 24, art. 5470, pp. 1-10, Dec. 2019.

[38]. Mazur M, Kaczmarek D, Domaradzki J, Wojcieszak D, Song S, and Placido F, "Influence of thickness on transparency and sheet resistance of ITO thin films," Presented in the Eighth International Conference on Advanced Semiconductor Devices and Microsystems, 2010, pp. 6568.

[39]. Wang HF, Ritter T, Cao WW, and Shung KK, "High frequency properties of passive materials for ultrasonic transducers," IEEE Trans. Ultrason. Ferroelectr., Freq. Control, vol. 48, no. 1, pp. 7884, Jan. 2001. [PubMed: 11367809]

[40]. Schellenberg MW and Hunt HK, "Hand-held optoacoustic imaging: A review," Photoacoustics, vol. 11, pp. 14-27, Sep. 2018. [PubMed: 30073147] 

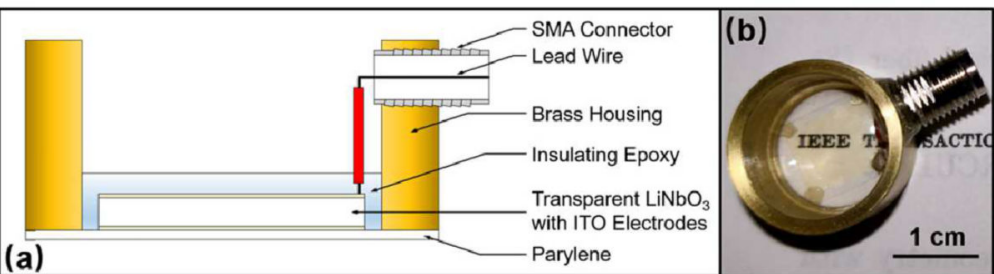

Fig. 1.

(a) Design cross section of the transparent ultrasonic transducer (not to scale); (b) photograph of a fabricated transparent ultrasonic transducer.

(a)

Parylene 
(a)

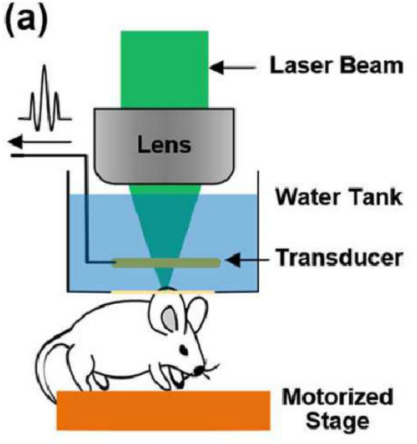

(b)

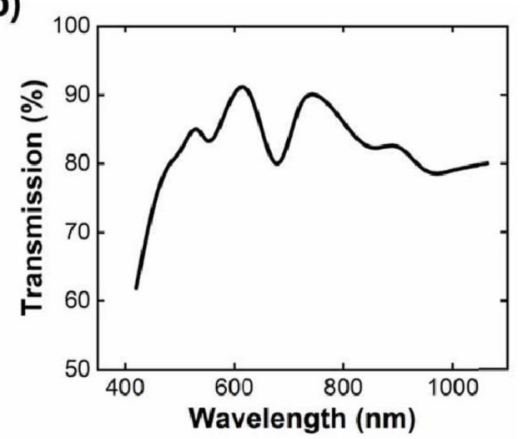

Fig. 2.

(a) Schematic of the reflection-mode PAM. The transparent transducer, directly in the optical path, is placed on the top of the imaging object to acquire the excited PA waves. The imaging object was mounted on a motorized stage for raster scans; (b) Measurement of optical transmission through the fabricated transducer. 

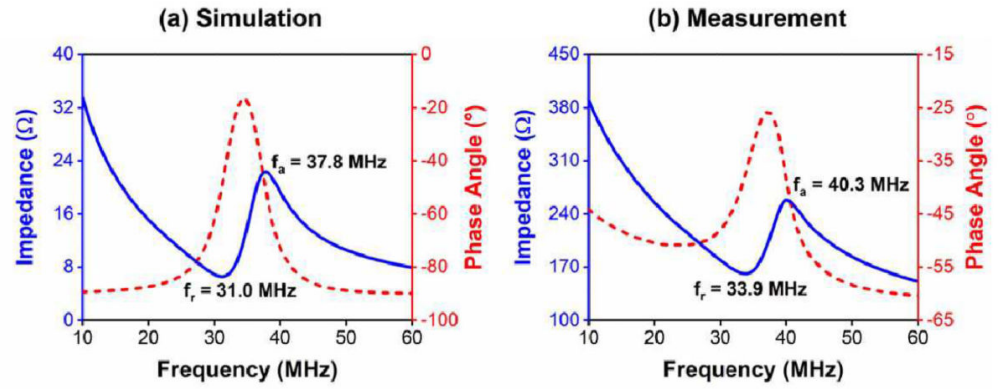

Fig. 3.

(a) PiezoCAD simulated and (b) measured electrical impedance (blue) and phase angle (red) of a transparent transducer. 
(a) Simulation

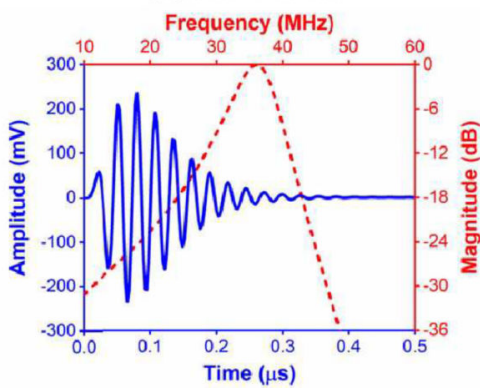

(b) Measurement

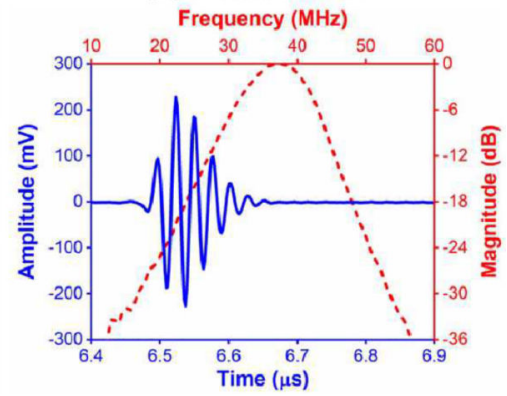

Fig. 4.

(a) PiezoCAD simulated and (b) measured pulse-echo response waveform (blue) and its frequency spectrum (red) of a transparent transducer. 


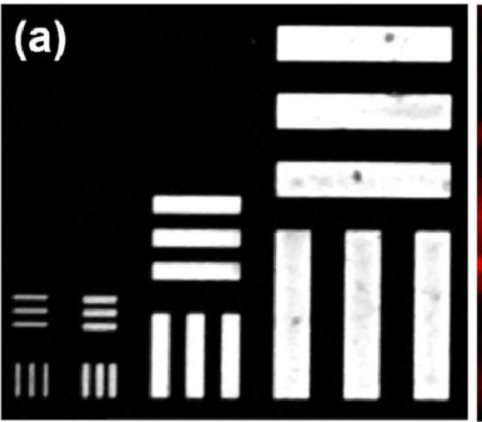

Norm. PA amplitude 0

\section{(b)}

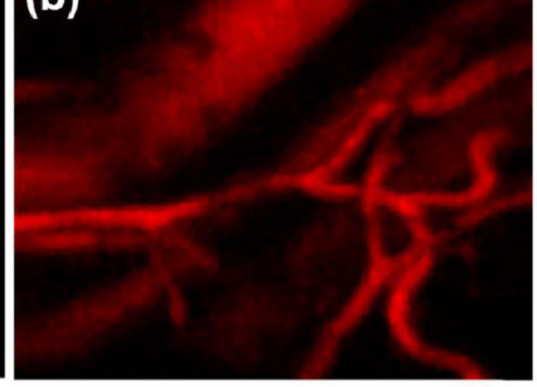

Norm. PA amplitude

$100 \mu \mathrm{m}$

Fig. 5.

(a) MAP image of the carbon-nanotube pattern. (b) MAP image of an area in the mouse ear showing the vasculatures by exciting the hemoglobin in RBCs. This imaging data was averaged 16 times. 
TABLE I

Design Parameters of the Transparent High-frequency Ultrasonic Transducer.

\begin{tabular}{cc}
\hline Specifications & Values \\
\hline Designed center frequency & $37 \mathrm{MHz}$ \\
Aperture size & $1 \mathrm{~cm} \times 1 \mathrm{~cm}$ \\
Thickness of piezoelectric material $\left(\mathrm{LiNbO}_{3}\right)$ & $100 \mu \mathrm{m}$ \\
Thickness of matching layer (parylene) & $16 \mu \mathrm{m}$ \\
Thickness of backing (EPO-TEK 301) & $1 \mathrm{~mm}$ \\
\hline
\end{tabular}

Specifications

ned center frequency

$\frac{\text { }}{\frac{1}{2}}$ 